\title{
Occupational therapy interventions in pulmonary rehabilitation - an update in the COVID-19 ERA
}

\section{CIUBEAN Alina Deniza ${ }^{1}$, CIORTEA Viorela Mihaela ${ }^{1,2}$, UNGUR Rodica Ana $^{1,2}$, BORDA Ileana Monica ${ }^{1,2}$, DOGARU Bombonica Gabriela ${ }^{1,2}$, POPA Theodor ${ }^{2}$, IRSAY Laszlo ${ }^{1,2}$}

Editor: Constantin Munteanu, Romanian Association of Balneology, office@bioclima.ro

Reviewers: Silișteanu Sînziana Călina and Rotariu Mariana

*Corresponding author: CIORTEA Viorela Mihaela, E-mail: viorela.ciortea@yahoo.com

1. University of Medicine and Pharmacy "Iuliu Hațieganu", Cluj-Napoca, Romania

2. Clinical Rehabilitation Hospital, Cluj-Napoca, Romania

\begin{abstract}
Background. As symptoms of COVID-19 infection are varying in severity and type, the long-term disability is yet to be established due to a short time-window since the pandemic started. Most survivors will have persistent pulmonary symptoms even after the infection, which raises the awareness of the importance of pulmonary rehabilitation in these patients, as they are mostly young, with severely diminished quality of life as they are unable to perform their basic activities of daily living as before. Occupational therapy is a form of rehabilitation treatment aimed at maximizing functionality and independence in performing activities of daily living, improvement of the patient's autonomy and prevention of further functional decline.

Objective. The purpose of the current work is to review the most important occupational therapy interventions applicable during a pulmonary rehabilitation program for chronic pulmonary pathologies, that can also be applied in COVID-19 survivors with persistent respiratory symptoms.

Discussion. The main objectives of occupational therapy in pulmonary rehabilitation are training using breathing techniques at rest and during task performance, upper limbs training to increase exercise tolerance, programming and simplifying daily activities, informing patients of the importance of asking for help, planning the day/week, organizing the environment, educating the patient. All these objectives can be achieved in a simple way and at low-cost.

Conclusions. Occupational therapy intervention during comprehensive pulmonary rehabilitation must be promoted to specifically evaluate and solve problems related to respiratory disability. Occupational tasks should be related to symptoms occurring during specific activities. Standardized protocols and definition of outcomes during occupational therapy intervention are lacking.
\end{abstract}

Keywords: occupational therapy, pulmonary rehabilitation, COVID-19, activities of daily living, quality of life,

\section{INTRODUCTION}

On March 11 2020, coronavirus disease 2019 (COVID-19) was declared a global pandemic by the World Health Organization (WHO). COVID-19 is caused by the coronavirus SARS-CoV-2 and can present with a wide spectrum of clinical symptoms. The long-term issues experienced by survivors of COVID-19 after discharge are yet to be established, but previous coronavirus outbreaks of severe acute respiratory syndrome (SARS) in 2002 and Middle East respiratory syndrome (MERS) in 2012 could be helpful in predicting postdischarge symptoms, as it was found that one-quarter of survivors of SARS and MERS had reduced lung function and exercise capacity at 6 months postdischarge (1).

In the COVID-19 era, the importance of pulmonary rehabilitation (PR) has arised. Until 2020, most rehabilitation guidelines dealt with chronic pulmonary conditions. Among the variety of pulmonary conditions, the most disabling are the chronic pathologies, like chronic obstructive pulmonary disease (COPD) and idiopathic pulmonary fibrosis (IPF) (2). COPD is a multifactorial progressive chronic lung disease that causes airflow obstruction, that results in persistent and progressive breathlessness, productive coughing, fatigue and recurrent chest infection (3). COPD is sometimes associated with extrapulmonary disorders such as muscle wasting, osteopenia, cardiovascular disease and depression (4). IPF is a chronic progressive disorder with a poor prognosis (5). The hallmark symptom is progressive dyspnea, frequently accompanied by a nonproductive cough, that causes exercise limitation. The presence of dyspnea and exercise limitation lead to difficulties in performing activities of daily living (ADLs) or instrumental activities of daily living (IADLs) and contribute to impairments in the patients' quality of life (QoL) (6).

As symptoms of COVID-19 infection are varying in severity and type, the long-term disability is yet to be established due to a short time-window since the pandemic started. Some studies have concluded that most 
survivors will have persistent pulmonary symptoms even after the infection, which raises the awareness of the importance of PR in these patients as they are mostly young, with severely diminished QoL as they are unable to perform their basic ADLs or IADLs as before. A recent report on postdischarge symptoms and rehabilitation needs in survivors of COVID-19 infection in the United Kingdom reported extremely high levels of fatigue, breathlessness, persistent symptoms relating to communication, voice, swallow, and laryngeal sensitivity, problems in mobility, self-care or usual activities. More importantly, the duration of symptom persistence appears to be greater than that seen in community-acquired bacterial pneumonia

Additionally, a study conducted on an Italian population of 143 individuals at 7 weeks after hospital discharge found that most subjects still experience fatigue, breathlessness and joint pain (8).

$\mathrm{PR}$ is an important component in the management of chronic pulmonary pathologies, as it is proven to significantly relieve dyspnea and fatigue, improve emotional function and enhance the sense of control that individuals have over their condition, being overall beneficial in improving QoL and exercise capacity (9). Thus, the goals of PR are to stabilize or reverse the disease process, alleviate symptoms, restore functional capacities as much as possible, reduce disability, and enhance QoL. A multidisciplinary rehabilitation team working with the patient can design an individualized treatment program to meet this end (10).

Occupational therapy (OT) is a form of rehabilitation treatment aimed at maximizing functionality and independence in performing activities of daily living (ADLs), instrumental ADLs (IADLs), improvement of the patient's autonomy and prevention of further functional decline. When OT is part of PR, the outcome for patients with chronic respiratory impairment is improved (11). Occupational therapists are rehabilitation professionals who work to help individuals engage in ADLs and other meaningful "occupations" that affect their health, well-being, and participation in life roles $(12,13)$.

OT goals in PR are patient centered and patient driven and includes ADL evaluation and training to increase functional endurance, instruction and training in appropriate breathing techniques, evaluation and strengthening of the upper extremity, instruction for work simplification and energy conservation, evaluation of the need for adaptive equipment, assistance in adapting leisure activities, education in stress management and relaxation techniques (12-14).

Pulmonary patients usually report a certain degree of fatigue while performing most ADLs. During simple tasks, like "combing hair" or "tying shoes", chronic pulmonary patients tend to develop a breathing pattern: rapid, irregular shallow breathing during the activity and rapidly and deeply afterwards. This is due to the rapid and ineffective shallow breathing during the bending and arm muscle activity, resulting in compensatory hyperventilation (15).

Additionally, other relatively simple tasks, like sweeping, changing a light bulb, lifting a pot or erasing a blackboard result in 50\%-60\% oxygen consumption pf the maximal oxygen uptake and increases the minute ventilation, which can explain the sensation of dyspnea and physical discomfort in pulmonary patients. When tasks become more intense, walking while carrying weights or climbing stairs, the dyspnea increases (16).

\section{Objective}

Considering that most pulmonary patients experience certain symptoms while performing ADLs or IADLs, the overall QoL is severely diminished. At the present time, the long-term effects of COVID-19 can only be speculated, but most survivors deal with persistent respiratory symptoms, like fatigue and dyspnea, which can be also managed by a comprehensive PR program that should include OT. As these symptoms are similar to those in patients dealing with COPD or IPF, there are no limits into using the techniques we have in order to increase QoL in these patients post-infection. The lack of knowledge about OT roles and benefits may be hampering its inclusion in PR programs and preventing people with respiratory diseases from getting the best evidence-based care.

Thus, the purpose of the current work is to review the most important OT interventions applicable during a PR program for chronic pulmonary pathologies like COPD or IFD, that can also be applied in COVID-19 survivors with persistent respiratory symptoms.

\section{Discussion}

The main objectives of OT in PR are training diaphragmatic breathing at rest and during task performance, upper limbs training to increase exercise tolerance, programming and simplifying daily activities, informing patients of the importance of asking for help, planning the day/week, organizing the environment, educating the patient. All these objectives can be achieved in a simple way and at low-cost (17).

\subsection{ADL evaluation and training using breathing techniques}

Patients with chronic pulmonary conditions are often limited in their ability to perform their ADLs mostly due to the presence of dyspnea, which is the most obvious sign that an individual is having difficulty breathing. In its most severe form, the patient is short of breath at rest and is not able to utter a short phrase without gasping for air. Often, patients with COPD hold their breath, breathe shallowly and fast, or elevate their shoulders as they breathe. Associated with dyspnea, the patient can experience extreme fatigue, cough, confusion, impaired 
judgement (18).

The therapist should observe and note the patient's breathing pattern during the ADL evaluation and should identify the precipitating factors (e.g., "Mr. F. becomes short of breath when washing his face while seated in front of the sink"). Also, the oxygen saturation (O2Sat) with activity should also be measured by pulse oximetry, and if it falls below $90 \%$ as the patient performs basic ADLs, the use of oxygen with certain activities should be considered. If the patient does not have home oxygen, the physician should be informed in order to prescribe oxygen supplementation at home. Also, as part of the functional assessment, measurements of heart rate and blood pressure should also be taken (19). Pausing an activity should occur if $\mathrm{SpO} 2$ drops below target or Borg scale dyspnea score $>3$ with consideration of breathing technique like pursed lip breathing with resumption of exercise intervention once $\mathrm{SpO} 2$ reaches target $(20,21)$. Breathlessness can be reduced in patients with COPD by teaching them to adopt dyspnea control postures. For example, when sitting, the patient should slightly bend forward at the waist while supporting the upper body by placing the forearms on the table or on his/hers thighs. When standing, leaning forward and propping the body on a counter or shopping cart may help with the issue $(22,23)$.

It is important that OT specialists teach the patient breathing techniques to use during the performance of ADLs. Pursed-lip breathing prevents tightness in the airway by providing resistance to expiration. This technique is performed by a nasal inspiration followed by expiratory blowing against pursed lips to decrease airway collapse, reduce respiratory rate and dynamic hyperinflation during exercise training with the aim of an overall increase endurance (23). It increases the use of the diaphragm and decreases accessory muscle recruitment (24). Instructions for pursed-lip breathing are the following: (a) purse your lips as if you are going to whistle; (b) slowly exhale through pursed lips-you should feel some resistance; (c) inhale deeply through your nose; and (d) it should take you twice as long to exhale as it does to inhale. After learning pursed lip breathing, the patient should use these breathing techniques while performing tasks that previously caused them to be breathless $(23,24)$.

Also, timing the breath during an activity could also be helpful. For example, the patient should breathe out while pushing the vacuum cleaner and breathe in while pulling the vacuum cleaner. Moreover, exhaling when lifting an object puts less pressure not only on the lungs but also on the cardiovascular system as it prevents the Valsalva maneuver (25).

Another technique that stimulates the use of the diaphragm to improve chest volume is diaphragmatic breathing. The technique can be taught with the patient positioned in a comfortable position, like sitting, semifowlers position (sitting at a $45^{\circ}$ angle), side-lying, or sitting with trunk flexion. The OT specialist should position the pelvis (posterior pelvic tilt), neck (extension), eyes (upward) and upper and lower extremities (external rotation and flexion). Moreover, providing external stimulus can facilitate the technique. For example, placing one hand of the patient on the abdomen near the umbilicus and the other on the sternal manubrium and instruct him to observe the increasing of the abdomen and decreasing of the chest while the therapist should loudly inhale and exhale alongside the patient. The patient is asked to "breathe into your hand" while inhaling through the nose and exhaling orally with pursed lips (26). An easier method is by placing a small paperback novel on the abdomen just below the thorax. The person lies supine and is instructed to inhale slowly and make the book rise. Exhalation through pursed lips should cause the book to fall.

IADLs include activities that support daily life and enable an individual to successfully live life to its fullest and interact with his/her environment and community. Examples of IADLs include home management, shopping, meal preparation, driving and community mobility, pet care, financial management, medication management, care of others, leisure tasks, employment, education, rest/ sleep, and social participation. The OT may provide recommendations to modify tasks or alter the environment to reduce extraneous effort and decrease activity demand. Energy conservation techniques are commonly embedded into IADL re-training. Examples of recommendations include making larger meals to freeze, letting dishes air dry, grouping task items together to minimize unnecessary searches, sliding rather than carrying items, shopping with someone who can carry grocery bags, or using grocery home delivery services (27).

As individuals with COPD or IFD experience dyspnea mostly during the performance of an activity, another intervention should be made into managing dyspnearelated anxiety. This can be achieved by monitoring and adjusting their breathing in a controlled therapeutic environment and implementing dyspnea control postures, paced activity, and breathing techniques while ensuring safe performance of the activity (28).

Active cycle of breathing techniques can be used to ventilate obstructed lung areas. Autogenic drainage can be used to mobilize and centralize secretions with short breaths to collect secretions in the peripheral airways, followed by normal breaths to collect secretions into the intermediate airways, and deep breaths and huff cough to expel secretions $(29,30)$. A huff cough is performed with an open glottis as it creates an increase in the linear velocity of the expiratory airflow and propels secretions. By initiating a forced expiration at a low lung volume the 
equal pressure point is moved to the periphery and small airways, while a forced expiration from a high lung volume will move the equal pressure point centrally towards the large central airway (31).

Additionally, posturing the patient plays an important role in respiratory function, and it is effective, simple, and easy to accomplish. Positioning should be used over other techniques like postural drainage given the pathophysiology of COVID-19 and the observed V/Q mismatch (32-34). Patients should be encouraged to adopt, whenever possible, a sitting and standing position to maximize lung function, increase lung compliance and elastic recoil, shift mediastinal structures and provide mechanical advantage in forced expiration (35).

PR or breathing exercises should be stopped if chest pain, palpations, and dizziness occur, or if $\mathrm{SpO} 2$ does not recover and the patient is unable to maintain Borg scale dyspnea score below 4, with rest and oxygen supplementation (36).

\subsection{Upper Extremity Function}

Pulmonary patients are often treated with steroids, have systemic inflammation, are older and hypoxic, and therefore often have muscle weakness. Patients with COPD commonly use the accessory muscles of the shoulder girdle to help them breathe, making it difficult for them to use these muscles while conducting an unsupported upper extremity activity (37). Many patients with COPD report disabling dyspnea for daily activities involving the upper extremities like lifting objects or grooming at work levels much lower than for lower extremity exercises $(38,39)$.

The main objective in training the upper extremity is to increase exercise tolerance. Patients should be taught strategies to program their activities with distinct levels of demand. They should always start with light and slow activities, such as personal hygiene from a sitting position, with support of the upper extremity while brushing teeth, combing hair, shaving, applying make-up, and continue with those that can't be performed with the upper extremity supported, like showering or armpit shaving (40).

Upper extremity strengthening has been found to improve the quality of life by increasing the capacity to work and reducing the oxygen requirement of upper extremity activity. Use of free weights, Theraband $\AA$, an arm ergometer, and other upper body strengthening techniques are all helpful in increasing upper body strength. Additional improvement in functional status is seen when leg training is added $(41,42)$.

\subsection{Work Simplification and Energy \\ Conservation}

Fatigue, shortness of breath, and limited endurance are common factors that may limit performance and participation. OT strategies should be aimed at modifying tasks and making recommendations regarding the use of assisted devices and/or adaptive equipment to reduce effort associated with the performance of daily routines. Work and/or ADL performance capacity are significantly reduced in patients with COPD or IPF, and they should benefit from instruction in work simplification and energy conservation. Energy conservation techniques are tools that aim at reducing the energy expenditure during the performance of ADLs, decreasing the sensation of dyspnea and increasing the functionality (43).

These interventions should include simple strategies such as eliminating unnecessary steps, sitting versus standing if possible, setting up task equipment in advance to minimize effort and using lightweight tools or utensils. Additionally, patients are encouraged to pace themselves through activities and take rest breaks prior to experiencing fatigue (44).

Bathing is a particularly strenuous activity as the hot humid air makes breathing difficult, which is why it is recommended to use a ventilation fan or leave the door open while bathing to keep the humidity level down. Also, the use of a chair in the shower and a thick terry robe after showering instead of toweling off are two suggestions that are helpful in reducing energy expenditure.

Also, unsupported UE activity is very fatiguing, and the patients should be taught to support their arms during certain UE activities such as hair combing or shaving. Also, as mentioned above, scheduling of activities that require more energy expenditure for the time following the use of a bronchodilator will also allow patients to accomplish more.

As the pulmonary disease progresses, some adaptive equipment can be useful. Because bending over to tie shoes or put on pants may cause significant shorthness of breath, elastic shoe laces, a long-handled shoehorn, or a reacher to assist with putting on slacks may be helpful.

Simplifying task performance by adapting the environment should also be included in the OT interventions: elevation of the toilet seat, hand rails in the bathroom or bedroom, long-handled shoe horns, walkers with seats and bags etc (45).

\subsection{Stress and Anxiety Management}

Stress and anxiety are common by-products of respiratory disorders. Due to the fact that COVID-19 has caused a public emergency, patients with COVID-19 may demonstrate different degrees of psychological disorders, such as anger, fear, anxiety, depression, insomnia, and loneliness (46). Providing education to help individuals manage their shortness of breath is an important step in lessening anxiety and promoting participation in the treatment program. Interventions include strategies to help clients prioritize activities and create a balanced lifestyle, increase awareness of body and mind interaction to manage breathing, increase confidence to manage stressors and perform daily activities with more 
confidence, and provide education on a variety of relaxation methods. These could include progressive muscle relaxation techniques, pursed-lip breathing technique, and diaphragmatic breathing (47).

Additionally, teaching patients methods to cope with extreme shortness of breath can lessen their fear. Leaning forward and resting their arms on the table releases the diaphragm and makes breathing easier. Using pursed lip and/or active expiration helps to slow the pace of breathing so that the patient is not breathing shallowly and rapidly.

Also, a stress management technique such as visualization may help patients calm themselves by mentally transporting them out of the stressful situation. It is important that the patient practice these options prior to actually needing them. Having a well-practiced plan of action for the panic associated with breathlessness will give patients confidence in their ability to control the situation (48).

\section{Conclusions}

The three major components of the medical system are prevention, treatment and rehabilitation, all being equally important. OT intervention during comprehensive PR must be promoted to specifically evaluate and solve problems related to respiratory disability. Occupational tasks should be related to symptoms occurring during specific activities. Standardized protocols of OT during PR and definition of outcomes during OT intervention are lacking.

\section{Funding}

This research did not receive any specific grant from funding public, commercial, or not-for-profit agencies.

\section{Author contribution}

All authors have consistently contributed to this article.

\section{Declaration of interests}

This article does not contain any studies with human or animal subjects. This study did not require written

consent from patients. The authors declare that they have no known competing financial interests or personal relationships that could have appeared to influence the work reported in this paper.

\section{References}

1. Ahmed H, Patel K, Greenwood DC, Halpin S, Lewthwaite P, Salawu A, Eyre L, Breen A, O'Connor R, Jones A, Sivan M. Long-term clinical outcomes in survivors of severe acute respiratory syndrome (SARS) and Middle East respiratory syndrome (MERS) coronavirus outbreaks after hospitalisation or ICU admission: a systematic review and meta-analysis. Journal of rehabilitation medicine. 2020 May 5;52(5):1-1.

2. Pauwels RA, Buist AS, Calverley PM, Jenkins CR, Hurd SS. Global strategy for the diagnosis, management, and prevention of chronic obstructive pulmonary disease: NHLBI/WHO Global Initiative for Chronic Obstructive Lung Disease (GOLD) Workshop summary. American journal of respiratory and critical care medicine. $2001 \mathrm{Apr}$ 1;163(5):1256-76.

3. Agusti AG. COPD, a multicomponent disease: implications for management. Respiratory medicine. 2005 Jun $1 ; 99(6): 670-82$.

4. Agusti AG, Noguera A, Sauleda J, Sala E, Pons J, Busquets X. Systemic effects of chronic obstructive pulmonary disease. European Respiratory Journal. 2003 Feb $1 ; 21(2): 347-60$.

5. Kekevian A, Gershwin ME, Chang C. Diagnosis and classification of idiopathic pulmonary fibrosis. Autoimmunity reviews. 2014 Apr 1;13(4-5):508-12.

6. Swigris JJ, Kuschner WG, Jacobs SS, Wilson SR, Gould MK. Health-related quality of life in patients with idiopathic pulmonary fibrosis: a systematic review. Thorax. 2005 Jul 1;60(7):588-94.

7. Halpin SJ, McIvor C, Whyatt G, Adams A, Harvey O, McLean L, Walshaw C, Kemp S, Corrado J, Singh R, Collins T. Postdischarge symptoms and rehabilitation needs in survivors of COVID-19 infection: A cross-sectional evaluation. Journal of medical virology. 2021 Feb;93(2):1013-22.

8. Carfî A, Bernabei R, Landi F. Persistent symptoms in patients after acute COVID-19. Jama. 2020 Aug 11;324(6):603-5.

9. McCarthy B, Casey D, Devane D, Murphy K, Murphy E, Lacasse Y. Pulmonary rehabilitation for chronic obstructive pulmonary disease. Cochrane database of systematic reviews. 2015(2).

10. Griffiths TL, Burr ML, Campbell IA, Lewis-Jenkins V, Mullins J, Shiels K, Turner-Lawlor PJ, Payne N, Newcombe RG, Lonescu AA, Thomas J. Results at 1 year of outpatient multidisciplinary pulmonary rehabilitation: a randomised controlled trial. The Lancet. 2000 Jan 29;355(9201):362-8.

11. Lorenzi CM, Cilione C, Rizzardi R, Furino V, Bellantone T, Lugli D, Clini E. Occupational therapy and pulmonary rehabilitation of disabled COPD patients. Respiration. 2004;71(3):246-51

12. Snyder N, Wilson R, Finch L, Gallant B, Landa C, Frankel D, Brooks D, Packham T, Oliveira A. The Role of Occupational Therapy in Pulmonary Rehabilitation Programs: Protocol for a Scoping Review. JMIR research protocols. $2021 \mathrm{Jul}$ 26;10(7):e30244.

13. Sewell L. Occupational therapy and pulmonary rehabilitation. In: Clini E, Holland AE, Pitta F, Troosters T, editors. Textbook of Pulmonary Rehabilitation. Cham, Switzerland: Springer International Publishing; Feb 2018:159-169.

14. Easthaugh S, Bradley G, Peel L, Donnelly J. Occupational therapy-led pulmonary rehabilitation: A practice analysis. British Journal of Occupational Therapy 2019 Sep 19;82(12):770-774.

15. Tangri, S; Wolf, CR. The breathing pattern in Chronic Obstructive lung Disease during the performance of some common daily activities. Chest. 1973;63:126-7.

16. Velloso M, Stella SG, Cendon S, Silva AC, Jardim JR. Metabolic and ventilatory parameters of four activities of daily living accomplished with arms in COPD patients. Chest. 2003 Apr 1;123(4):1047-53. 
17. Ogden LD, Deveene C. COPD program guidelines for occupational therapists and other health professionals. O'Dell-Rossi P, Browning G, Barry J. Occupational therapy in pulmonary rehabilitation. Maryland: Ramsco. 1985:50-8.

18. Garvey C, Bayles MP, Hamm LF, Hill K, Holland A, Limberg TM, Spruit MA. Pulmonary rehabilitation exercise prescription in chronic obstructive pulmonary disease: review of selected guidelines. Journal of cardiopulmonary rehabilitation and prevention. 2016 Mar 1;36(2):75-83.

19. Karnani N, Reisfield G, Wilson GR. Evaluation of chronic dyspnea. American family physician. $2005 \mathrm{Apr}$ 15;71(8):1529-37.

20. Sharp JT, Drutz WS, Moisan T, Foster J, Machnach W. Postural relief of dyspnea in severe chronic obstructive pulmonary disease. American Review of Respiratory Disease. 1980 Aug;122(2):201-11.

21. O'Neill S, McCarthy D. Postural relief of dyspnoea in severe chronic airflow limitation: relationship to respiratory muscle strength. Thorax. 1983 Aug 1;38(8):595-600.

22. Rodriguez-Morales AJ, Cardona-Ospina JA, GutiérrezOcampo E, et al. :. Clinical, laboratory and imaging features of COVID-19: A systematic review and metaanalysis. Travel Med Infect Dis. March 2020:101623.

23. Mayer AF, Karloh M, Dos Santos K, de Araujo CLP, Gulart AA. Effects of acute use of pursed-lips breathing during exercise in patients with COPD: a systematic review and meta-analysis. Physiotherapy. 2018;104:9-17.

24. Breslin EH. The pattern of respiratory muscle recruitment during pursed-lip breathing. Chest. 1992 Jan 1;101(1):75-8.

25. Dolmage TE, Janaudis-Ferreira T, Hill K, Price S, Brooks D, Goldstein RS. Arm elevation and coordinated breathing strategies in patients with COPD. Chest. 2013 Jul $1 ; 144(1): 128-35$.

26. Cahalin LP, Braga M, Matsuo Y, Hernandez ED. Efficacy of diaphragmatic breathing in persons with chronic obstructive pulmonary disease: a review of the literature. Journal of Cardiopulmonary Rehabilitation and Prevention. 2002 Jan 1;22(1):7-21.

27. Christiansen $\mathrm{CH}$. Defining lives: Occupation as identity: An essay on competence, coherence, and the creation of meaning. American Journal of Occupational Therapy. 1999 Nov 1;53(6):547-58.

28. Migliore A. Management of dyspnea guidelines for practice for adults with chronic obstructive pulmonary disease. Occupational therapy in health care. 2004 Jan 1;18(3):1-20.

29. McKoy NA, Saldanha IJ, Odelola OA, Robinson KA. Active cycle of breathing technique for cystic fibrosis. Cochrane Database Syst Rev. 2012;12:CD007862.

30. McCormack P, Burnham P, Southern KW. Autogenic drainage for airway clearance in cystic fibrosis. Cochrane Database Syst Rev. 2017;10:CD009595.

31. McIlwaine M, Bradley J, Elborn JS, Moran F. Personalising airway clearance in chronic lung disease. Eur Respir Rev. 2017;26:160086.

32. Fink JB. Positioning versus postural drainage. Respir Care. 2002;47:769-777.

33. Gattinoni L, Coppola S, Cressoni M, Busana M, Chiumello D. Covid-19 Does Not Lead to a "Typical" Acute
Respiratory Distress Syndrome. Am J Respir Crit Care Med. March 2020.

34. Tang X, Du R, Wang R, et al. :. Comparison of Hospitalized Patients with Acute Respiratory Distress Syndrome Caused by COVID-19 and H1N1. Chest. March 2020:S0012369220305584.

35. Jones SE, Barker RE, Nolan CM, Patel S, Maddocks M, Man WDC. Pulmonary rehabilitation in patients with an acute exacerbation of chronic obstructive pulmonary disease. J Thorac Dis. 2018;10(S12):S1390-S1399.

36. Cullen DL, Rodak B. Clinical utility of measures of breathlessness. Respir Care. 2002;47:986-993.

37. Hodgkin JE, Celli BR, Connors GL, editors. Pulmonary rehabilitation: guidelines to success. Lippincott Raven; 2000.

38. Celli BR, Rassulo J, Make BJ. Dyssynchronous breathing during arm but not leg exercise in patients with chronic airflow obstruction. New England Journal of Medicine. 1986 Jun 5;314(23):1485-90.

39. Hair C. The breathing pattern in chronic obstructive lung disease during the performance of some common daily activities. Chest. 1973 Jan;63(1).

40. Nici L, Lareau S, ZuWALLACK RI. Pulmonary rehabilitation in the treatment of chronic obstructive pulmonary disease. American Family Physician. 2010 Sep 15;82(6):655-60.

41. Ries AL, Ellis B, Hawkins RW. Upper extremity exercise training in chronic obstructive pulmonary disease. Chest. 1988 Apr 1;93(4):688-92.

42. Ries AL. Pulmonary rehabilitation: summary of an evidence-based guideline. Respiratory care. 2008 Sep 1;53(9):1203-7.

43. Velloso M, Jardim JR. Functionality of patients with chronic obstructive pulmonary disease: energy conservation techniques. Jornal Brasileiro de Pneumologia. 2006 Dec;32(6):580-6.

44. Rashbaum I, Whyte N. Occupational therapy in pulmonary rehabilitation: energy conservation and work simplification techniques. Physical Medicine and Rehabilitation Clinics. 1996 May 1;7(2):325-40.

45. Mahoney K, Pierce J, Papo S, Imran H, Evans S, Wu WC. Efficacy of adding activity of daily living simulation training to traditional pulmonary rehabilitation on dyspnea and health-related quality-of-life. PloS one. 2020 Aug 27;15(8):e0237973.

46. Wang Y, Di Y, Ye J, Wei W. Study on the public psychological states and its related factors during the outbreak of coronavirus disease 2019 (COVID-19) in some regions of China. Psychology, health \& medicine. 2021 Jan $2 ; 26(1): 13-22$.

47. Courtney C, Escobedo B. A stress management program: Inpatient-to-outpatient continuity. American Journal of Occupational Therapy. 1990 Apr 1;44(4):306-10.

48. Paz-Díaz H, De Oca MM, López JM, Celli BR. Pulmonary rehabilitation improves depression, anxiety, dyspnea and health status in patients with COPD. American journal of physical medicine \& rehabilitation. 2007 Jan 1;86(1):30-6. 